\title{
Optimal Allocation of Complex Equipment System Maintainability
}

\section{Xin Ren}

Graduate School, National Defense University, Beijing, 100091, China

Medical Protection Laboratory, Naval Medical Research Institute, Shanghai, 200433, China

Email:renxins8410130163.com

\section{Yan Kong}

Machine Engineering Department, Naval Petty Officer Academy, Bengbu, 233012, China

Email:hgky@163.com

\section{Jiwen Cui}

Department of Military Logistics and Science and Technology Equipment, National Defense University, Beijing ,100091, China

Email: cjwa163. com

\section{Kai Zhang}

Medical Protection Laboratory, Naval Medical Research Institute, Shanghai, 200433, China

Email:zk@163.com

\section{Li Ma}

Medical Protection Laboratory, Naval Medical Research Institute, Shanghai, 200433, China

Email:ml@163. com

The maintainability allocation of equipment system is a multi-objective programming problem with multiple constraints. Based on the defects of traditional algorithm, it is difficult to conquer all the constraints at the same time, and it is difficult for the result to reflect different satisfaction levels on the targets. This paper proposes a method in combination with genetic algorithm (GA) and satisfaction function. Firstly, use GA to solve the optimal solution of the single target; secondly, get the satisfaction level of the total target through that of the single target; finally, obtain satisfactory solution of the problem with GA. Results show that the method can effectively solve the defects above, which is significant to the optimal allocation of equipment system maintainability. 


\section{Introduction}

The traditional maintainability allocation methods, mainly simple quantitative methods[1], is hard to apply to the highly complex equipment system. The maintainability allocation problem was studied when the minimum maintenance cost and the shortest maintenance time are satisfied at the same time[2]; however the models are with complex nonlinear characteristics, especially when the number of parts in equipment systems increases [3], the solution space will be exponentially increasing. It is difficult for the conventional method to obtain ideal optimization results (for example: the result can't meet the constraint conditions). In addition, the conventional method, in which the linear weighted method is used, finds it hard to give reasonable weighted coefficient in the multi-objective optimization; therefore, the paper introduces a method in combination with the genetic algorithm (GA) and the satisfaction function, and the simulation example shows its effectiveness.

\section{Maintainability Allocation Model of Equipment System}

(1) Model 1: the shortest maintenance time

$$
\min \bar{M}_{c t}=\frac{\sum_{i=1}^{n} \lambda_{i} M_{c t i}}{\sum_{i=1}^{n} \lambda_{i}}
$$

The constraints are:

$$
\begin{gathered}
\sum_{i=1}^{n}\left(k_{i}\left(\frac{1}{\lambda_{i} M_{c t i}}\right)+\lambda_{i} T_{i} C_{r i} M_{c t i}+\lambda_{i} T f_{i}\right) \leq C_{0} \\
L_{i} \leq M_{c t i} \leq M_{i}
\end{gathered}
$$

$\bar{M}_{c t}$ is the mean maintenance time, $M_{c t i}$ is the maintenance time of the ith part, $\lambda_{i}$ is the failure rate of the $\mathrm{i}^{\text {th }}$ part. Formula (2.2) indicates the upper limit of the maintenance cost. Formula (2.3) indicates the upper limit and the lower limit of the maintenance time of each part.

(2) Model 2: the minimum maintenance cost

$$
\min C=\sum_{i=1}^{n}\left(k_{i}\left(\frac{1}{\lambda_{i} M_{c t i}}\right)+\lambda_{i} T_{i}\left(C_{r i} M_{c t i}+f_{i}\right)\right) \leq C_{0}
$$

The constraints are: 


$$
\begin{gathered}
\frac{\sum_{i=1}^{n} \lambda_{i} M_{c t i}}{\sum_{i=1}^{n} \lambda_{i}} \leq \bar{M}_{c t}^{*} \\
L_{i} \leq M_{c t i} \leq M_{i}
\end{gathered}
$$

$C$ is maintenance cost. Formula (2.5) indicates the upper limit of the mean maintenance time. Formula (2.6) indicates the upper limit and the lower limit of the maintenance time of each part.

\section{Satisfaction Function of the Target}

The satisfaction function of the target takes the objective function as the independent variable, and utilizes the objective function's satisfaction level to represent the decision maker's satisfaction in the target value. 0 indicates that the result is the most satisfied to the decision maker, and 1 indicates the opposite meaning. Suppose that there be a programming model where minimum value is needed for calculation. The value of objective function $R$ is between $R_{\text {min }}$ and $R_{\min }$. When $R=R_{\min }$, the satisfaction level equals 1 , and When $R=R_{\max }$, the satisfaction level equals 0 .

In the multi-objective programming problems, the total satisfaction function is needed based on each target satisfaction function. Suppose that satisfaction functions of target 1 and target 2 be $h 1(R 1), h 2(R 2)$, and the total satisfaction function is hown as follows[4].

$$
h(R)=[h 1(R 1)]^{w 1} \wedge[h 2(R 2)]^{w 2}
$$

$w 1, w 2$ are the weighted coefficients, whose value interval are $[0,1]$ to, reflect the importance of different goals. 1 indicates that the goal is the most important, 0 indicates that the goal can be ignored, and $\wedge$ is an operator to select a smaller number.

\section{Calculation Method by Using Genetic Algorithm}

Genetic algorithm (GA) is a kind of adaptive optimization method, with many advantages such as smart search and gradual optimization [5]. Here we introduce the details of the method.

1) Encoding

Chromosome uses the binary coding. The maintenance time of each component is signified by a binary number containing 8 bits considering both the calculation precision and the speed, so the whole length of chromosomes is $8 n$. ( $n$ is the total number of components in the equipment system.) 
2)Fitness function

Use penalty factor to define the fitness function considering constraints of the optimization problem, seen in Type (4.1), which is the maximum objective function, and Type (4.2), which is the minimum objective function .

$$
\begin{aligned}
& f=\left\{\begin{array}{cl}
N-M & x \text { meets the constraints } \\
N+f(x) & x \text { can't meet the constraints }
\end{array}\right. \\
& f=\left\{\begin{array}{cl}
\frac{1}{N+M} & x \text { meets the constraints } \\
\frac{1}{N+f(x)} & x \text { can't meet the constraints }
\end{array}\right.
\end{aligned}
$$

$f$ is the fitness function, $f(x)$ is the objective function, $M$ is punishment factor, $N$ is an constant used to make sure that the fitness is positive. In the paper, $N=300, M=200$. When the model 1 and 2 are solved respectively, the fitness function is established according to Type (4.2), and the objective functions indicate the maintainability time, and the maintainability cost. When the two models are solved synthetically, the fitness function is established according to Type (4.1), and the objective function refers to the total satisfaction level, seen in Type (3.1) [6].

\section{Calculation and Analysis}

Supposing that a certain equipment system have five units, the upper limit for maintenance cost is 1.45 million and the upper limit for average maintenance time is 0.75 hour. In this algorithm, the population size is 5000, the biggest evolution times is 100 , the length of the chromosome is 40 , the initial crossover probability is 0.7 , the initial mutation probability is 0.1 .

\subsection{Calculation by using genetic algorithm}

Model 1 and Model 2 are calculated separately by using GA. To reduce the random impact on the results, run the program five times, and the results are shown in Fig. 1 and Fig. 2. From the two figures, we can see that the results are slightly different and tend to be convergent every time. 


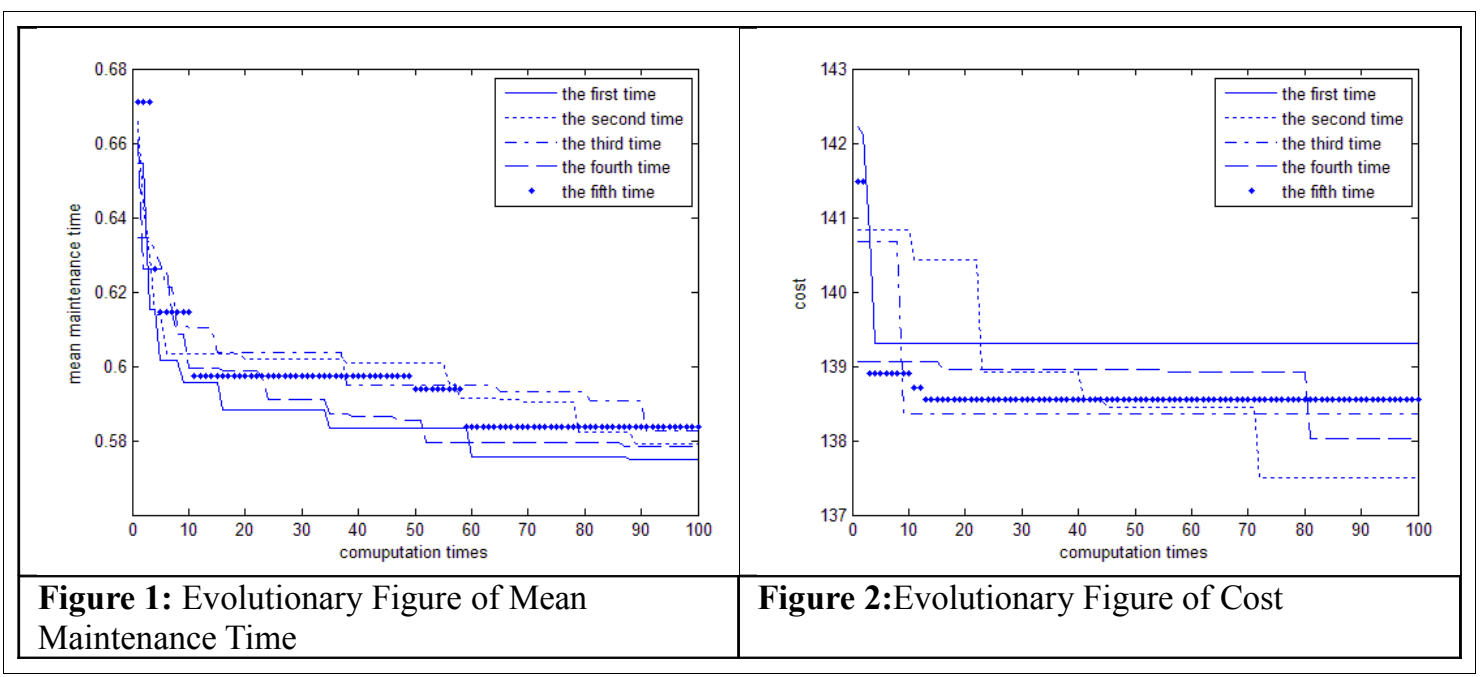

According to the results above, obtain the scopes of the two objectives that average maintenance time is $0.55 \sim 0.75$, and that maintenance cost is $134.6 \sim 145$; therefore, respectively establish the satisfaction of both functions (linear), as shown in (5.1 ) (5.2), the total satisfaction function according to the type structure (3.1).

$$
\begin{gathered}
h 1\left(\bar{M}_{c t}\right)=-5 \bar{M}_{c t}+3.75 \\
h 2(C)=-0.096 C+13.94
\end{gathered}
$$

\subsection{Comparison with other methods}

For comparison, Tables 1 and 2 respectively list the result of Lingo software and GA algorithm. It can be seen that, for a single goal programming model, the solution results of Lingo software can not meet the corresponding constraint conditions, and GA can solve the problem better.

For multi-objective programming model, compare the solution results when the weighted factors are the same. The results of Lingo software can not reflect the consistency between importance degrees of two goals, namely, the importance degree of Goal 1 is obviously above that of Goal 2. (The satisfaction level of goal 1 is 0.97 and the satisfaction level of goal 2 is 0.05.) We can conclude that, it is hard for weighted factors to adjust the importance degrees of different goals in the linear weighted method, while the results obtained from the satisfaction function is relatively more reasonable.

\begin{tabular}{|c|c|c|c|c|c|c|c|c|c|}
\hline & $\boldsymbol{M}_{c t 1}$ & $\boldsymbol{M}_{c t 2}$ & $\boldsymbol{M}_{c t 3}$ & $\boldsymbol{M}_{c t 4}$ & $\boldsymbol{M}_{c t 5}$ & $\boldsymbol{M}_{c t}$ & $\boldsymbol{C}$ & $\mathrm{h} 1$ & $\mathrm{~h} 2$ \\
\hline Model 1 & 0.50 & 0.42 & 1.0 & 1.83 & 0.80 & 0.56 & 144.84 & 1 & 0 \\
\hline Model 2 & 0.50 & 0.60 & 1.0 & 2.45 & 1.03 & 0.74 & 137.58 & 0 & 1 \\
\hline $\begin{array}{c}\mathrm{w} 1=0.5, \mathrm{w} 2=0 . \\
5\end{array}$ & 0.5 & 0.48 & 1.0 & 2.14 & 1.00 & 0.66 & 139.92 & 0.47 & 0.47 \\
\hline
\end{tabular}

Table 1 : Results by Using GA in Table 1 


\begin{tabular}{|c|c|c|c|c|c|c|c|c|c|}
\hline & $\boldsymbol{M}_{c t 1}$ & $\boldsymbol{M}_{c t 2}$ & $\boldsymbol{M}_{c t 3}$ & $\boldsymbol{M}_{c t 4}$ & $\boldsymbol{M}_{c t 5}$ & $\boldsymbol{M}_{c t}$ & $\boldsymbol{C}$ & $\mathrm{h} 1$ & $\mathrm{~h} 2$ \\
\hline Model 1 & 0.5 & 0.4 & 1.0 & 1.5 & 0.8 & 0.55 & 147 & 1 & 0 \\
\hline Model 2 & 0.5 & 0.8 & 1.0 & 2.5 & 1.7 & 0.94 & 134.6 & 0 & 1 \\
\hline$\alpha=0.5$ & 0.5 & 0.4 & 1.0 & 1.85 & 0.8 & 0.56 & 145 & 0.97 & 0.05 \\
\hline
\end{tabular}

Table 2 :Results by Using Lingo Software Solution

\section{Conclusion}

The paper studied the maintainability allocation model of equipment system by using GA and satisfaction function. Compared with the traditional methods, it solves the flaws that it is hard to fulfill the constraint conditions in the single objective programming and to reflect the significance difference of different goals. The method and conclusions can provide reference to the maintainability allocation of the equipment system.

\section{References}

[1] Jian-min Zhao, Hong Zhou. An optimal allocation method for system maintainability[J]. Mechanical science and technology. 19(2),219-220 (2000) (In Chinese)

[2] Yong Zhang, Xian-Hang Chao. A Study on maintenance optimal distribution of equipment system based on hereditary algorithm $[\mathrm{J}]$. East China electric power. 12(1),85-87(2003) (In Chinese)

[3] Guang-Chen Bai, Yan Chen. Satisfactory solution of multi-objective optimization of system reliability[J]. Systems engineering and electronics. 22(8),85-87(2000) (In Chinese)

[4] L. N. Xing, Y. W. Chen, Yang K W. A hybrid approach combining an improved genetic algorithm and optimization strategies for asymmetric traveling salesman problem $[\mathrm{J}]$. Engineering Applications of Artificial Intelligence, 2008,21(8):1370-1380.

[5]K. Kamall, L. J. Jiang, J. Yen, et al. Using Q-learning and genetic algorithms to improve the efficiency of weight adjustments for optimal control design problems[J]. Journal of Computing and Information Science in Engineering, 2007,7(4):302-308.

[6] L. N. Xing, Y. W. Chen, K. W. Yang,A hybrid approach combining an improved genetic algorithm and optimization strategies for asymmetric traveling salesman problem $[\mathrm{J}]$. Engineering Applications of Artificial Intelligence, 2008,21(8):1370-1380. 